\title{
Arts et éducation relative à l'environnement : un maillage incontournable
}

Vincent Bouchard-Valentine

\section{(2) OpenEdition}

Édition électronique

URL : http://journals.openedition.org/ere/2630

DOI : $10.4000 /$ ere.2630

ISSN : 2561-2271

Éditeur

Centr'ERE

\section{Référence électronique}

Vincent Bouchard-Valentine, « Arts et éducation relative à l'environnement : un maillage incontournable », Éducation relative à l'environnement [En ligne], Volume 14 - 1 | 2017, mis en ligne le 15 septembre 2017, consulté le 10 décembre 2020. URL : http://journals.openedition.org/ere/2630 ; DOI https://doi.org/10.4000/ere.2630

Ce document a été généré automatiquement le 10 décembre 2020. 


\title{
Arts et éducation relative à l'environnement : un maillage incontournable
}

\author{
Vincent Bouchard-Valentine
}

1 L'histoire fourmille d'exemples où le pouvoir mobilisateur et transformateur de l'art a servi différentes causes sociales, politiques, religieuses, voire commerciales. Pour les artistes, le lien entre arts et environnement a aussi donné lieu à des maillages féconds. Ce numéro thématique se penche sur la relation plus spécifique entre les arts et l'éducation relative à l'environnement (ERE). J'entends par "arts " les pratiques artistiques, bien sûr, mais également, et peut-être surtout, les démarches de médiation et d'éducation artistiques, étant donné que nous intervenons ici dans la sphère éducative. Ce maillage entre les arts et l'ERE n'est à mon avis valide et pertinent que si les deux champs contributifs préservent leurs visées respectives, à savoir l'approfondissement du rapport à l'environnement pour l'ERE et l'approfondissement $\mathrm{du}$ rapport au sensible au monde, pour les arts. En effet, dans ce projet qui nous interpelle, les arts ne devraient, en aucun cas, être subordonnés aux visées de l'ERE. Les deux champs devraient plutôt s'enrichir mutuellement à travers une démarche inédite - qu'il reste à formaliser - favorisant leur entrelacement, voire leur fusionnement, tout en préservant leurs finalités propres.

2 La prise en compte de la dimension esthétique du rapport à l'environnement apparaît comme un angle mort des travaux de recherche francophones en ERE ${ }^{1}$. Pourtant, l'environnement est un sujet de prédilection pour un nombre grandissant d'artistes et il se trouve, dans les pratiques d'éducation formelle et non formelle, de nombreuses initiatives qui pourraient aisément être rattachées à une proposition Arts \& $E R E^{2}$.

3 C'est ce contexte qui nous a amenés à repérer, dans la Francophonie, des chercheurs intéressés par les relations entre pratique artistique, médiation ou éducation artistiques et ERE. Certains d'entre eux ont répondu à l'appel du Centr'ERE pour une première session Arts \& ERE tenue lors du colloque Éducation et environnement : points de 
rencontre vers une transformation écosociale dans le cadre du $84^{\mathrm{e}}$ congrès de l'Association francophone pour le savoir (Acfas) en 2016, à l'Université du Québec à Montréal.

Par la suite, pour enrichir la documentation de recherche, nous avons souhaité réaliser un premier regroupement de textes francophones dans ce numéro thématique de la revue Éducation relative à l'environnement : Regards - Recherches - Réflexions. Il paraissait d'autant plus opportun de proposer cet ouvrage que ce champ de recherche naissant offre des perspectives théoriques et pédagogiques d'une grande richesse en cette période agitée qui appelle une mobilisation généralisée pour tenter de contrer la dégradation de l'écosystème planétaire.

Ce numéro paraît au moment où la Faculté des arts et la Faculté des sciences de l'éducation de l'Université du Québec à Montréal s'unissent pour célébrer le $50^{\mathrm{e}}$ anniversaire de la publication du rapport de la Commission d'enquête sur l'enseignement des arts dans la province de Québec (Rapport Rioux). Ce rapport de plus de 700 pages propose un véritable projet de société au cœur duquel les arts contribuent à la formation de citoyens libres et engagés dans l'édification d'une société véritablement humaniste. Or le projet Arts \& ERE porté par le Centr'ERE fait précisément écho aux visées sociales du Rapport Rioux, mettant en œuvre une démarche interdisciplinaire où interagissent le champ des arts et celui de l'éducation'.

6 Sans a priori, nous avons donc ouvert l'appel à contributions à un large registre d'objets d'études et de réflexions pour inciter le plus grand nombre de chercheurs et de praticiens réflexifs à proposer un texte s'inscrivant dans ce champ de recherche, au croisement de l'art, de l'éducation et de l'environnement, qui commence à se déployer en français. Les articles qui composent ce numéro ont été rédigés par des chercheurs établis ou émergents, des doctorants, des intervenants en contexte formel ou non formel, des artistes. L'hétérogénéité des thèmes abordés permet d'anticiper le foisonnement des projets pédagogiques et l'ampleur du champ de recherche que peut générer la mise en relation des multiples objets de l'ERE avec la diversité des pratiques artistiques d'ici, d'ailleurs et de toute époque. Trois idées fondamentales peuvent déjà être dégagées de l'analyse de ce corpus de textes.

\section{L'art, un creuset de redéfinition de notre rapport au monde}

7 La contribution des arts à l'ERE ne peut être réduite aux seules esthétiques dites « vertes » ou « écologiquement engagées ». L'héritage artistique de l'humanité doit être considéré dans son ensemble, puisqu'il représente la plus formidable source de connaissances dont nous disposons pour comprendre l'expérience humaine dans sa relation au monde.

8 Beaucoup d'artistes contemporains abordent des questions environnementales dans leurs œuvres et certains adoptent même une posture ouvertement militante pour la préservation et la restauration de l'environnement naturel. L'art écologiquement engagé rejoint bien entendu les visées de l'ERE. Il serait toutefois réducteur de limiter les expériences esthétiques en ERE à ce courant. Comme le souligne à juste titre Maia Morel, plusieurs pratiques artistiques contemporaines interpellent la société sur des enjeux d'actualité qui nécessitent une redéfinition de nos rapports à soi, aux autres et au monde. L'auteure pose son regard sur le Toutou de La Vérendrye, une mise en scène 
«apparemment spontanée» de peluches aux abords de la route nationale 117. Cette œuvre surprenante, qui invite la population à y contribuer, ne semble présenter qu'un intérêt ludique au premier abord. À regarder de plus près, on s'aperçoit cependant qu'elle questionne notre rapport à la nature, nos valeurs, nos attitudes et nos comportements individuels ou collectifs à l'égard de l'environnement. En ce sens, elle pourrait servir d'appui à une démarche d'ERE autour, par exemple, de la protection des milieux naturels, démarche qui pourrait à son tour alimenter un projet de création artistique collectif.

Le texte de Gina Thésée s'inscrit dans un tout autre registre artistique, celui des danses traditionnelles africaines qui entrent en dialogue «dans, avec, par et pour l'environnement». Cette auteure attire notre attention sur le fait que le corps est généralement évacué dans le traitement des questions socio-écologiques; symptôme de la cassure de notre lien avec la nature. N'est-ce pas dans le corps, nous rappelle-t-elle, que prennent naissance, se déploient et se cristallisent les multiples rapports à l'environnement: pulsions, sensations, perceptions, émotions, sentiments, pensées, conscience, paroles, mouvements, actions? Les danses d'inspiration africaine sont " une ode à la mort et la naissance, à la sécheresse et la pluie, à la moisson et la récolte, à la souffrance et la joie, à l'esclavage et la libération, à l'hostilité et l'amour ». En prenant appui sur ces danses, sur « leur corporéité, leur théâtralité, leur historicité et leur mondialité », la démarche d'ERE se réconcilie un peu avec un corps trop de fois agressé et blessé.

10 Magali Babin nous conduit hors de la nature et nous propose d'écouter les sons de la ville à travers des balades sonores immersives qui sont l'occasion d'explorer les relations entre l'être humain, l'espace sonore physique et l'espace sonore psychique. L'écoute y est envisagée comme « une expérience esthétique et sensible du réel dirigée vers les sphères environnementale, sociale et privée ». Pour Magali Babin, la balade sonore devient une démarche artistique pluridisciplinaire à travers laquelle l'artiste adopte la posture d'un apprenant critique de son environnement sonore : « Le bruit des grandes villes, ce murmure incessant, possède une certaine uniformité, au diapason des moteurs, même s'il se module selon les heures et parfois, aux rythmes des saisons. Ce bourdonnement citadin peut-il aussi se singulariser, ou bien ses qualités acoustiques sont-elles devenues universelles? » L'auteure suggère une pédagogie de l'écoute qui intensifie et consolide « la relation sensible entre l'individu et son environnement ».

11 Sylviane Blanc-Maximin et Angela Barthes présentent quant à elles deux dispositifs participatifs d'art contextuel ayant été implantés dans des territoires ruraux du sud de la France. Elles explicitent la filiation entre l'ERE et les principes de l'art contextuel, qui «s'empare de questions locales et globales pour solliciter le public à développer un regard critique ». L'étude qu'elles ont menée s'intéresse "à la réception des œuvres d'art contextuel par les acteurs non-artistes (habitants et participants du workshop) » et à «l'impact de ces projets en termes d'éducation relative au territoire ».

\section{L'art, une dimension incontournable d'une ERE holistique}

12 C'est sans conteste sur le plan d'une éducation holistique que la contribution des arts à l'ERE est la plus singulière et, à mon avis, la plus porteuse. Dans les théories contemporaines qui fondent les curricula scolaires, l'esthétique est en effet reconnue 
comme l'un des principaux modes de signification et d'appréhension du réel, au même titre que les mathématiques, les langues, les sciences ou la philosophie. Une conception holistique de l'ERE devrait donc intégrer la dimension esthétique du rapport à l'environnement pour favoriser chez l'apprenant des expériences significatives, ressenties dans les retranchements les plus intimes et subjectifs de son être.

Malgré plus de 50 ans d'un plaidoyer insistant et d'actions éducatives à petite et à grande échelle, le mouvement de l'ERE n'a visiblement pas encore atteint tous ses objectifs. Plus que jamais, l'actualité nous rappelle les agressions perpétrées contre la biodiversité et les écosystèmes à travers la planète. Le jeu dangereux auquel se livre l'humanité risque bientôt de rompre l'équilibre écologique actuel de la biosphère et d'entraîner le vivant dans un cycle de perturbations profondes dont les conséquences sont imprévisibles, mais sans aucun doute dramatiques. Les récents cris d'alarme lancés par des collectifs de scientifiques sont peut-être les derniers appels à la raison avant le point de non-retour. Nous sommes maintenant placés devant l'impérieuse et urgente nécessité d'un changement drastique de nos modes de vie.

Cette toile de fond menaçante met en lumière la nécessité de recourir à des stratégies éducatives transformatrices opérantes, car les approches rationnelles et culpabilisatrices n'ont visiblement pas réussi à mobiliser les populations qui semblent même avoir développé, au fil du temps, une "résistance " aux messages de ce type. Il est d'ailleurs assez troublant de constater que, dans les pays développés disposant de systèmes d'éducation avancés, une partie importante de la population ignore encore ou même réfute les évidences scientifiques qui attribuent aux activités humaines les dérèglements du climat.

Le texte d'Édith Planche aborde de front cette question fondamentale en faisant ressortir cette propension de l'épistémologie rationaliste à dissocier l'individu de son environnement, à ignorer le lien sensible qui l'unit au monde et à produire l'illusion qu'il peut y évoluer sans égard à la nature dont il est issu et qui assure sa survie. Pour Édith Planche, l'art est un "vecteur de liens avec le monde " qui permet de renouer avec une pensée holistique. La démarche artistique invite à «établir des liens avec l'environnement ", à " se mettre à l'écoute de sa propre résonance au monde » et à " habiter le paysage avec une partie de soi-même ».

C'est justement ce que cherche à instaurer Samuel Montigné par les randonnées de création art nature qu'il anime dans la forêt du mont Royal. Il aborde l'ERE par les sens, l'affectivité, la symbolique et l'intuition en amenant les randonneurs à exprimer leurs sensations, impressions et valeurs dans des dessins et des œuvres éphémères in situ. Cette dynamique particulière de création en nature favorise "une connexion plus harmonieuse à soi, au milieu et aux autres ». Pour cet auteur, «l'art nous propose une approche dynamique, existentielle, holistique et positive, qui restaure la confiance en soi et amène à revoir le fondement de nos valeurs».

17 Virginie Boelen articule quant à elle un projet éducatif dans le cadre d'un camp d'été pour enfants qui met en œuvre une approche écoformatrice expérientielle. L'aménagement d'un petit jardin devient l'occasion d'engager ces enfants dans différentes activités artistiques axées sur le dialogue avec le milieu de vie biotique. L'approfondissement du rapport sensible à l'environnement par l'art stimule ici le développement de l'empathie envers le vivant et de la pensée critique. 
Enfin, la démarche de création peut également être transférée en ERE pour résoudre des problèmes localisés. Diane Pruneau, Boutaina ElJai, Abdellatif Khattabi, Sara Benbrahim et Joanne Langis ont accompagné durant six mois des victimes d'inondations récurrentes au Maroc. Ces inondations, aggravées par les changements climatiques, polluent les sources d'eau potable, dévastent l'agriculture et les infrastructures routières, et causent différents problèmes de santé à la population qui les éprouve. À travers une démarche de pensée design, dix femmes ont cherché des moyens pour protéger leur famille et leurs biens. Elles ont analysé les différents aspects du problème, conçu et mis à l'essai différentes solutions pour remédier au manque d'eau potable en période d'inondation. Dans ce projet d'accompagnement, la démarche d'ERE est soutenue par des activités artistiques orientées vers la production et la mise en place de moyens de survie ayant un impact direct sur le bien-être des habitants de cette région.

\section{L'ERE, une contribution à l'expérience esthétique}

19 Si les arts peuvent enrichir l'ERE, cette dernière peut réciproquement enrichir la démarche de création artistique. Joanne Clavel développe par exemple depuis quelques années un modèle inédit de médiation scientifique par la danse où les savoirs scientifiques, particulièrement les savoirs écologiques, sont symbolisés par le mouvement. L'expérience de création chorégraphique est alors nourrie par une démarche d'apprentissage impliquant des scientifiques spécialistes de l'objet d'étude. L'auteure relate ici un projet chorégraphique vécu au Conservatoire professionnel de danse de Lugo en Espagne autour d'enjeux écologiques contemporains. Les élèves sont amenés à transposer en mouvements non seulement les savoirs scientifiques, mais aussi leur ressenti à l'égard des réalités écologiques qui leur sont associées. Ils « incarnent » en quelque sorte par la danse leur rapport à la problématique écologique étudiée : représentations, impressions, valeurs, attitudes, etc.

20 Liliane Dionne, Emerson Ferreira Gomes et Luís Paulo de Carvalho Piassi utilisent les arts pour créer un "tiers espace» entre la culture des jeunes et celle de leurs enseignants, afin d'accroître la signifiance des apprentissages scientifiques. Les élèves sont amenés à rendre compte des apprentissages scientifiques réalisés à travers des dessins, des sculptures et des poèmes ou encore à utiliser leur esprit critique scientifique pour apprécier des œuvres musicales ou cinématographiques. Les auteurs établissent également un parallèle entre les motivations des artistes et celles des scientifiques en rappelant les travaux de Robert Root-Bernstein qui considère l'esthétique comme un agent déterminant dans les avancées scientifiques.

21 Enfin, Anne Deslauriers effectue un retour sur le projet d'arts plastiques Marée d'Art Marée Noire qu'elle a piloté en milieu scolaire. Pour cette auteure, l'éducation contemporaine ne peut éluder la prise en compte des enjeux socio-écologiques qui confrontent nos sociétés. Elle s'appuie sur le Programme de formation de l'école québécoise et ses domaines généraux de formation pour concevoir des propositions de création artistique autour de tels enjeux et pour intégrer une démarche d'ERE à ses projets pédagogiques en art. La dimension éthique ajoutée aux propositions de création nourrit l'ensemble de la démarche et les œuvres produites témoignent des réflexions des élèves, de leurs visions du monde et des valeurs qui les habitent. Anne Deslauriers souligne que les productions des élèves sont souvent empreintes d'espoir et anticipent 
" un monde pouvant reprendre vie grâce à la puissance de leurs rêves ». Pour cette enseignante chercheure, ce type de projets donne un sens aux apprentissages artistiques.

\section{Conclusion}

Nous espérons que ce numéro thématique de la revue Éducation relative à l'environnement : Regards - Recherches - Réflexions contribuera à la consolidation et à l'extension d'un réseau de chercheurs et de praticiens intéressés par les relations entre pratique artistique, médiation et éducation artistiques, et éducation relative à l'environnement. Tel que signalé, pour soutenir ce projet, le Centr'ERE prévoit la mise en place d'un espace de convergence Arts \& ERE qui nous permettra de réunir les travaux de chercheurs de la Francophonie et d'autres espaces culturels, et de faire connaître les initiatives exemplaires susceptibles d'inspirer autant les intervenants, les chercheurs que les artistes. Nous invitons d'ores et déjà tous les artistes, enseignants et autres éducateurs qui souhaitent s'engager dans une telle démarche à se joindre à nous.

\section{NOTES}

1. Si la littérature anglophone propose un noyau relativement consistant d'écrits regroupés sous la vignette "Art-based Environmental Education", les perspectives francophones sont peu présentes dans la documentation de recherche.

2. Depuis l'idéation de ce numéro de la revue à l'automne 2016, le Centr'ERE a inscrit à son plan d'action l'axe de recherche Arts \& ERE, que je dirige avec Maia Morel, professeure en didactique des arts plastiques à l'Université du Québec en Abitibi-Témiscamingue.

3. Pour plus de détails sur les célébrations du Rapport Rioux : www.rapport-rioux.uqam.ca

\section{AUTEUR}

\section{VINCENT BOUCHARD-VALENTINE}

Professeur au Département de musique de l'Université du Québec à Montréal 